\title{
PMP(Porphyrin-Micelle-PSMA) Nanoparticles for Photoacoustic and Ultrasound Signal Amplification in Mouse Prostate Cancer Xenografts
}

\author{
Daehyun Kim ${ }^{1,2,3,+}$, Wonkook Han ${ }^{4,+}$, Jin Ho Chang $4, *$ (D) and Hak Jong Lee ${ }^{1,2,3,5, *(D)}$ \\ 1 Department of Nano Science and Technology, Graduate School of Convergence Science and Technology, \\ Seoul National University, Seoul 08826, Korea; daehyun.kim@nanoimgt.com \\ 2 Department of Radiology, Seoul National University Bundang Hospital, 82 Gumi-ro 173, Bundang-gu, \\ Seongnam 13620, Korea \\ 3 IMGT Co., Ltd., Seongnam 13605, Korea \\ 4 Department of Information and Communication Engineering, Daegu Gyeongbuk Institute of Science \\ and Technology (DGIST), Daegu 42988, Korea; wkhan@dgist.ac.kr \\ 5 Bio-MAX Institute, Seoul National University, 1 Gwanak-ro, Gwanak-gu, Seoul 08826, Korea \\ * Correspondence: jhchang@dgist.ac.kr (J.H.C.); hakjlee@snu.ac.kr (H.J.L.); Tel.: +82-53-785-6330 (J.H.C.); \\ +82-31-994-7077 (H.J.L.) \\ + These authors contributed equally to this work.
}

Citation: Kim, D.; Han, W.; Chang, J.H.; Lee, H.J. PMP(Porphyrin -Micelle-PSMA) Nanoparticles for Photoacoustic and Ultrasound Signal Amplification in Mouse Prostate Cancer Xenografts. Pharmaceutics 2021, 13, 1636. https://doi.org/ 10.3390/pharmaceutics13101636

Academic Editor: Jongbum Seo

Received: 1 September 2021

Accepted: 29 September 2021

Published: 7 October 2021

Publisher's Note: MDPI stays neutral with regard to jurisdictional claims in published maps and institutional affiliations.

Copyright: (c) 2021 by the authors. Licensee MDPI, Basel, Switzerland. This article is an open access article distributed under the terms and conditions of the Creative Commons Attribution (CC BY) license (https:/ / creativecommons.org/licenses/by/ $4.0 /)$.

\begin{abstract}
Photoacoustic (PA) imaging is used widely in cancer diagnosis. However, the availability of PA agents has not made great progress due to the limitations of the one currently in use, porphyrin. Porphyrin-Micelle (PM), developed by synthesizing porphyrin and PEG-3.5k, confirmed the amplification of the PA agent signal, and added binding affinity in an LNCaP model by attaching prostate-specific membrane antigen PSMA. Compared to the previously used porphyrin, a superior signal was confirmed, and the potential of PMP was confirmed when it showed a signal superior to that of hemoglobin at the same concentration. In addition, in the in vivo mouse experiment, it was confirmed that the signal in the LNCaP xenograft model was stronger than that in the PC-3 xenograft model, and the PMP signal was about three times higher than that of PM and porphyrin.
\end{abstract}

Keywords: photoacoustic; ultrasound; porphyrin; micelle; prostate cancer; cancer diagnosis; PSMA targeting

\section{Introduction}

Prostate cancer is the second-most common cancer in men worldwide [1]. The 5-year survival rates are relatively high, allowing numerous treatment options depending on the patient's condition [2]. Active surveillance, where cancer progression is monitored without intervention, is regularly exercised during the early stages, while interventionist treatment options such as chemotherapy, radiation, hormone therapy, and radical prostatectomy are also practiced to prevent disease progression $[3,4]$. Nevertheless, these interventions often have serious side effects such as urinary incontinence and erectile dysfunction, affecting the quality of life for those receiving the treatment [5-7]. As such, theranostic options with early diagnosis and minimal side effects are preferred [8]. Currently, biopsies, prostate-specific antigen (PSA) tests, ultrasound, and magnetic resonance imaging (MRI) are used for prostate cancer diagnosis. Each method has its limitations: biopsies are often invasive and may cause discomfort; PSA tests and ultrasound imaging are prone to misdiagnosis and often require biopsy confirmation; MRI imaging, while accurate, can be quite costly and is often used to complement the abovementioned methods [9-15]. As such, a minimally invasive, cost-efficient method to detect the disease in the early stages is strongly desired [16-18].

Porphyrin-based substances are currently being used in clinical practice [19-23]. For example, Visudyne is a porphyrin-based photosensitizer that is used to remove abnormal 
blood vessels in those with eye conditions such as macular degeneration [24-26]. Furthermore, due to their excellent biocompatibility and unique optical properties, porphyrinbased molecules are being actively investigated preclinically and clinically for cancer theranosis $[27,28]$. Accordingly, the optical properties of porphyrins have been maximized by employing them in photoacoustic (PA) imaging, where the molecules are excited by a laser to emit specific echogenic signals that are detected by ultrasound transducers. As PA imaging is extremely sensitive and minimally invasive, it has become a strong candidate for prostate cancer diagnosis [29-31].

To address the need for novel theranostic methods, we are reporting porphyrin-based micelles targeting the prostate-specific membrane antigen (PSMA) [32-34]. Identification of disease-specific biomarkers and targeting strategies have also greatly improved treatment options by minimizing potential side effects [35-37]. Accordingly, PSMA is a well-established biomarker for advanced prostate cancer, as prostate tumors highly overexpress this antigen [38,39]. In addition to such active targeting, preparation of porphyrins in micelles would also enhance their stability and half-life in circulation, thereby greatly improving their therapeutic window by combining improved tumor accumulation capacities and active targeting methods. We were able to demonstrate the robustness of PA signals from the porphyrin micelles themselves, and their superior sensitivity and selectivity against PSMA-expressing tumors in a xenograft mouse model. All in all, we believe that the concept of porphyrin micelles may become a strong candidate for the next generation of theranosis in prostate cancer patients.

\section{Results}

\subsection{Schematic of Porphyrin-Micelle-PSMA (PMP) Tumor Binding Phenomenon}

A summary of PMP mechanisms is described in Figure 1. As depicted in the figure, PSMA in PMP nanoparticles determines the intensity of binding affinity in PC-3 and LNCaP cancer cells. In the case of PC-3 which does not have PSMA binding site, since the binding affinity of PMP is relatively low, the phenomenon of materials passing in the direction of the arrow increases. Conversely, in the case of LNCaP with PSMA binding site, the amount of accession to cancer cells increases due to the effect of PSMA attached to PMP.

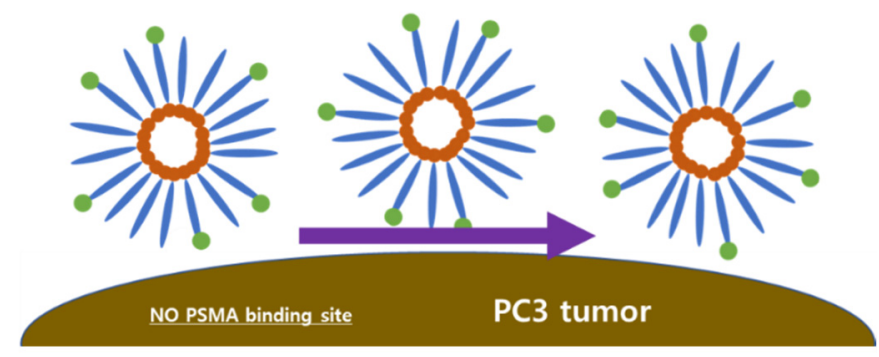

PMP - PC3 affinity

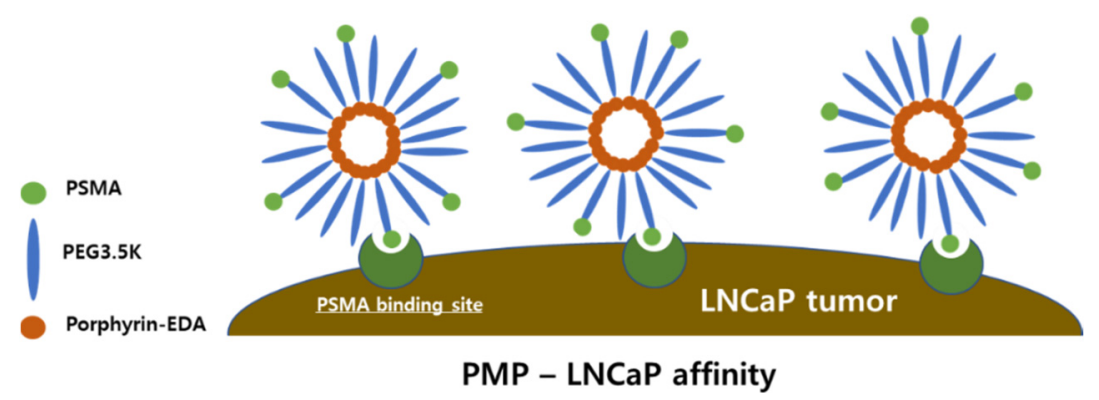

Figure 1. Schematic of PMP binding to the LNCaP tumor model. 


\subsection{Characterization of $P M$ and $P M P$}

Porphyrin-Micelle (PM) and Porphyrin-Micelle-PSMA (PMP) nanoparticles were characterized according to their size and zeta potentials (Table 1). For these measurements, dynamic light scattering was used (Malvern Zetasizer Nano, Malvern Instrument Ltd., Malvern, UK). There were no significant differences between the sizes and the total yields of the nanoparticle formulations, which had a mean diameter of $23 \pm 4.5$ and $26 \pm 6.2 \mathrm{~nm}$ for PM and PMP, respectively. The sizes and the shapes of the nanoparticles were also evaluated with scanning electron microscopy (SEM) and transmission electron microscopy (TEM) (Figure 2B). We confirmed the spherical shape of the nanoparticles and that they were well-dispersed across the medium. Furthermore, there was no statistical difference between the zeta potential values for PM and PMP, which had voltages of $-11.3 \pm 2.1$ and $-14.4 \pm 2.8 \mathrm{mV}$, respectively. As such, we speculated that the effects of the conjugated PSMA-targeting moiety on zeta potentials would be marginal. Furthermore, we examined the molecular weight of PM and PMP using MALDI-TOF, according to which data (Figure 2A), an increase in molecular weight was observed, suggesting the formation of Porphyrin Micelles. There was also an intensity between 3000 and $4000 \mathrm{~m} / \mathrm{z}$ in the PEG3.5K graph, and it was speculated that most sizes of PEG3.5K would be in this range. After PPR was conjugated to PEG3.5K, the $\mathrm{m} / z$ intensity in that range significantly decreased, because, we speculated, the PPR reacted better with the short PEG.

Table 1. Size and zeta potential of porphyrin micelle nanoparticles.

\begin{tabular}{ccc}
\hline Porphyrin Micelle & Size (nm) & Zeta Potential (mV) \\
\hline PM & $23 \pm 4.5$ & $-11.3 \pm 2.1$ \\
\hline PMP & $26 \pm 6.2$ & $-14.4 \pm 2.8$ \\
\hline
\end{tabular}

A

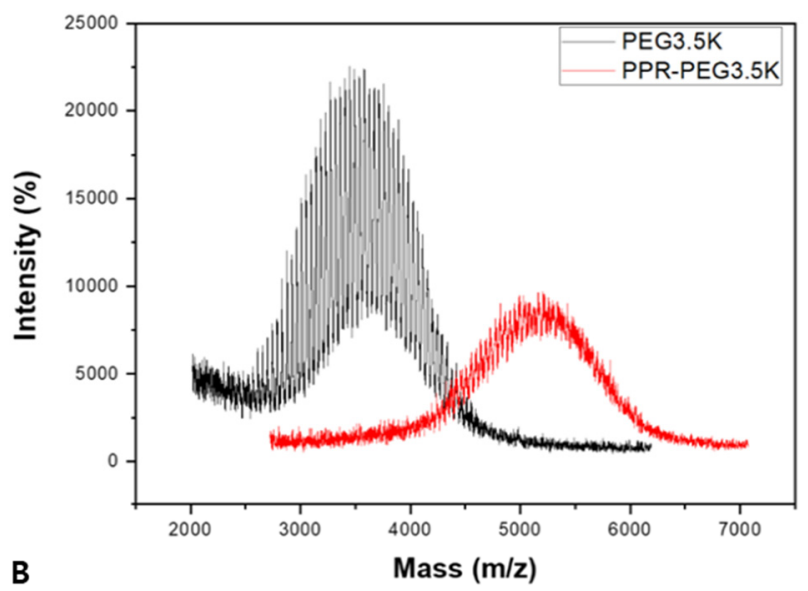

B

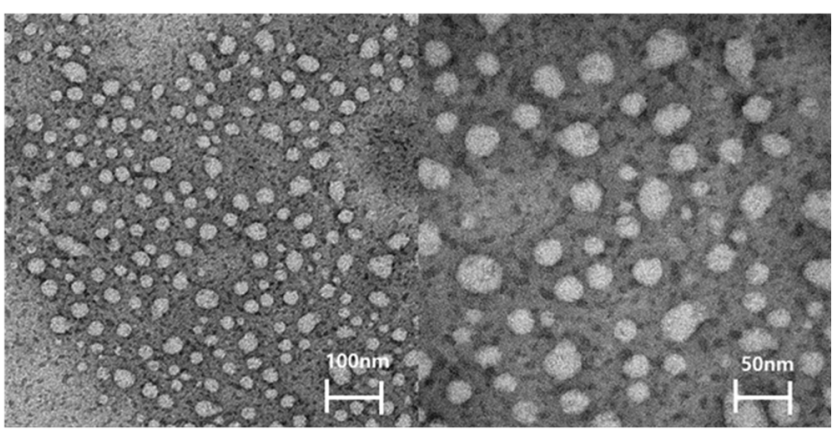

C

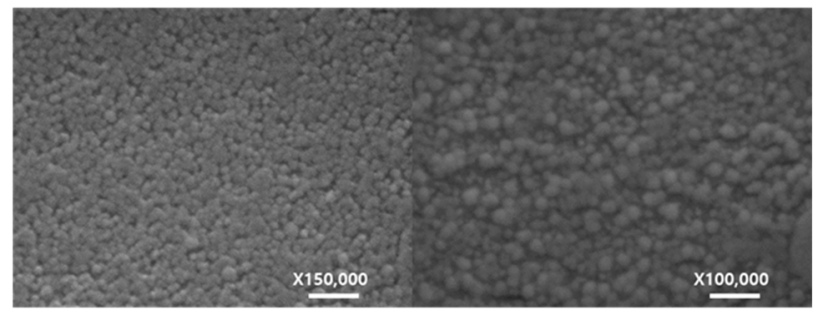

D

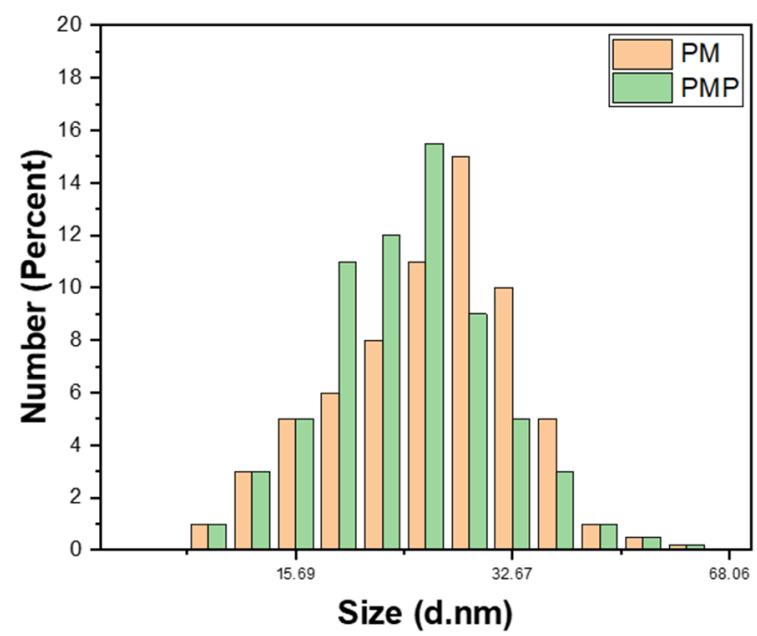

Figure 2. (A) MALDI-TOF data of PEG3.5K and porphyrin-PEG3.5K. (B) TEM image of PM. (C) SEM image of PM. (D) DLS data of PM \& PMP. 


\subsection{In Vitro Cell Viability Study and Confocal Microscopy}

The cytotoxic effects of Porphyrin, PM, and PMP were first evaluated in vitro using the PSMA-expressing LNCcP and PSMA-null PC3 cancer cell lines. First, different concentrations of Porphyrin, PM, and PMP were incubated with the cells to observe the effects on the cell viability of the two cell lines. Compared to the untreated control, none of Porphyrin, PM, and PMP groups had a significant effect on the viability or morphology of the LNCaP (Figure 3A) and PC3 (Figure 3B) cells. Next, the cells were incubated with PMP for $24 \mathrm{~h}$ and then observed under a confocal microscope. While a strong localization signal from PMP was observed on the surface of PSMA-expressing LNCaP cells, no fluorescence was observed in the PSMA-null PC 3 cells incubated with PMP, demonstrating the strong binding efficiency of the PSMA-targeting PMPs (Figure 3C).

A

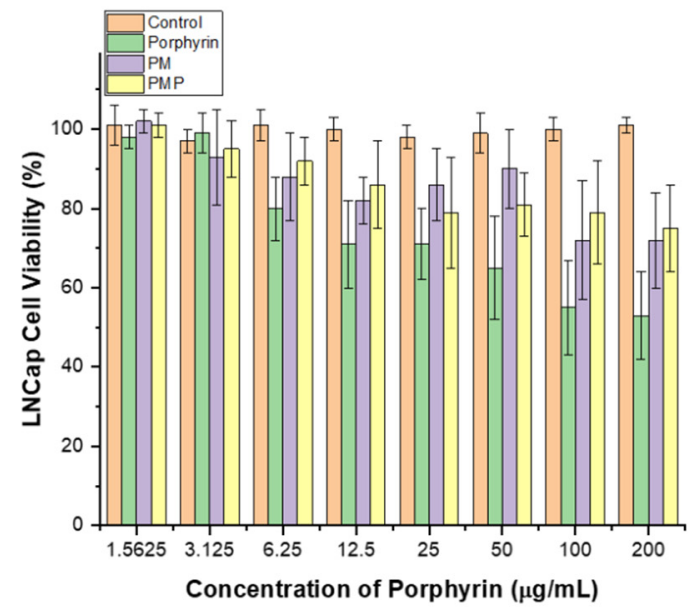

C

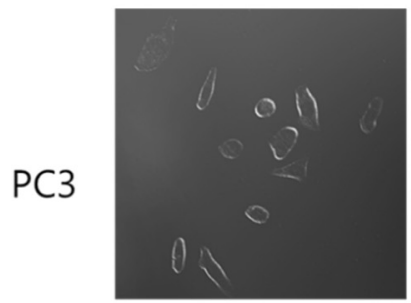

$\mathrm{DIC}$
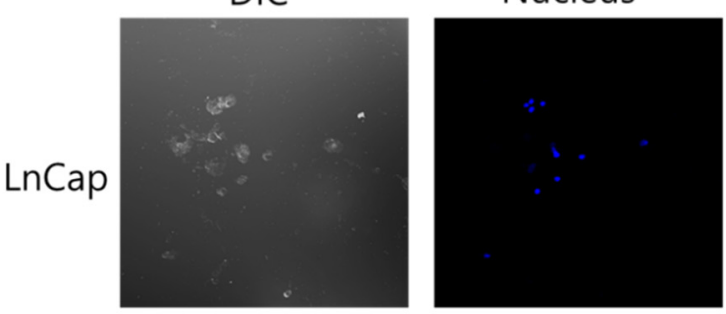

B
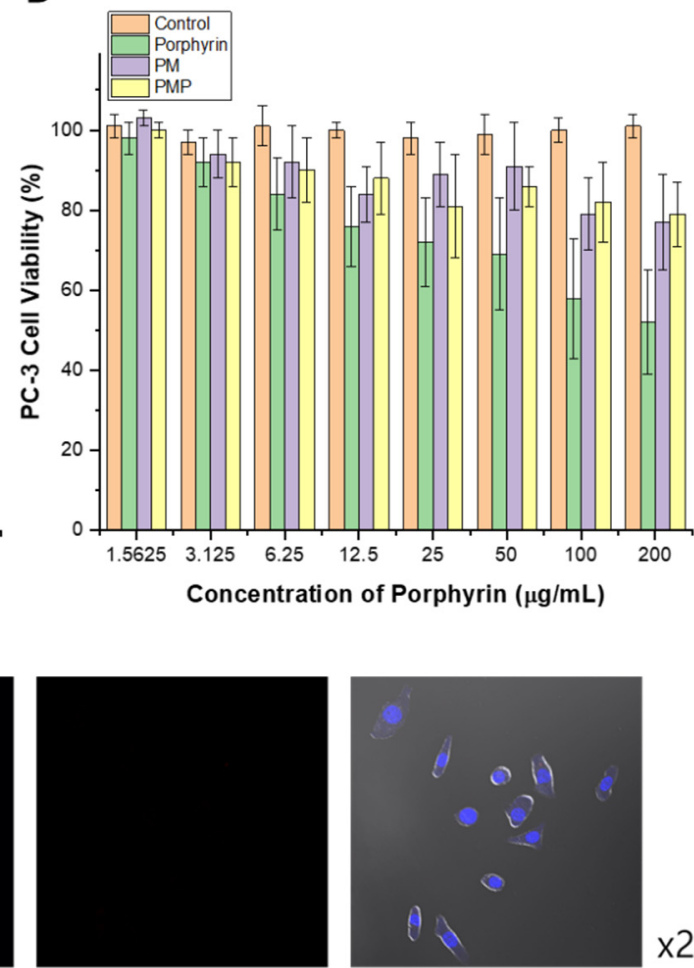

PPR-micelle

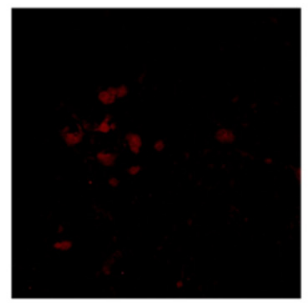

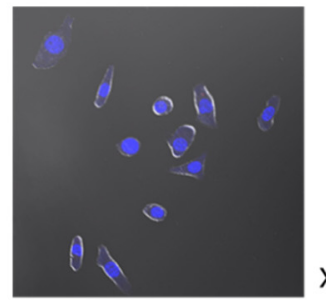

$x 200$

Merged

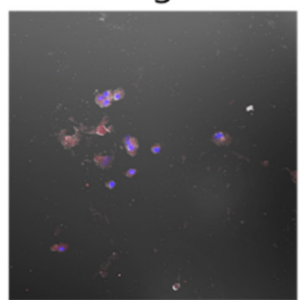

Figure 3. Cytotoxicity of porphyrin, PM, and PMP in vitro. (A) LNCaP cell line at difference material concentrations. (B) PC-3 cells at different material concentrations. (C) Confocal images of LNCaP and PC-3 cells treated with PMP to test binding affinity. Scale bar: $50 \mu \mathrm{m}$.

\subsection{Selection of Optimal Laser Wavelength}

For the selection of the optimal imaging laser wavelength, PA signals generated by porphyrin and PM were measured by changing the wavelength from 680 to $880 \mathrm{~nm}$ [40]. Note that changes in PA signal intensity were linearly proportional to the optical absorbance of a target. The ultrasound images in Figure 4A showed the upper and bottom portions of the silicone tube that contained PM nanoparticles, porphyrin, and water. Since the 
nanoparticle size was on the order of tens of nanometers and it was much smaller than the ultrasound wavelength (i.e., hundreds of micrometers), ultrasound backscattering was negligible and thus any information about the nanoparticles or porphyrin and water did not appear in the ultrasound images. In contrast, the PA images of PMP and porphyrin nanoparticles were clearly shown (Figure 4B) because the particles were able to absorb the laser energy and generate PA signals. Since the water did not contains any laser absorbers, low PA signals were observed. The average PA signal intensity was 2.89 times higher for PMP than for porphyrin (Figure 4C). This implies that the PMP particles were better laser absorbers than porphyrin. In addition, the PMP particles absorbed the laser to the maximum when the wavelength was $680 \mathrm{~nm}$; the PA signal generated by the PMP particles was 5.06 times higher than that of porphyrin at the same wavelength; thus, it was considered the optimal wavelength for PA imaging of the PMP particles.
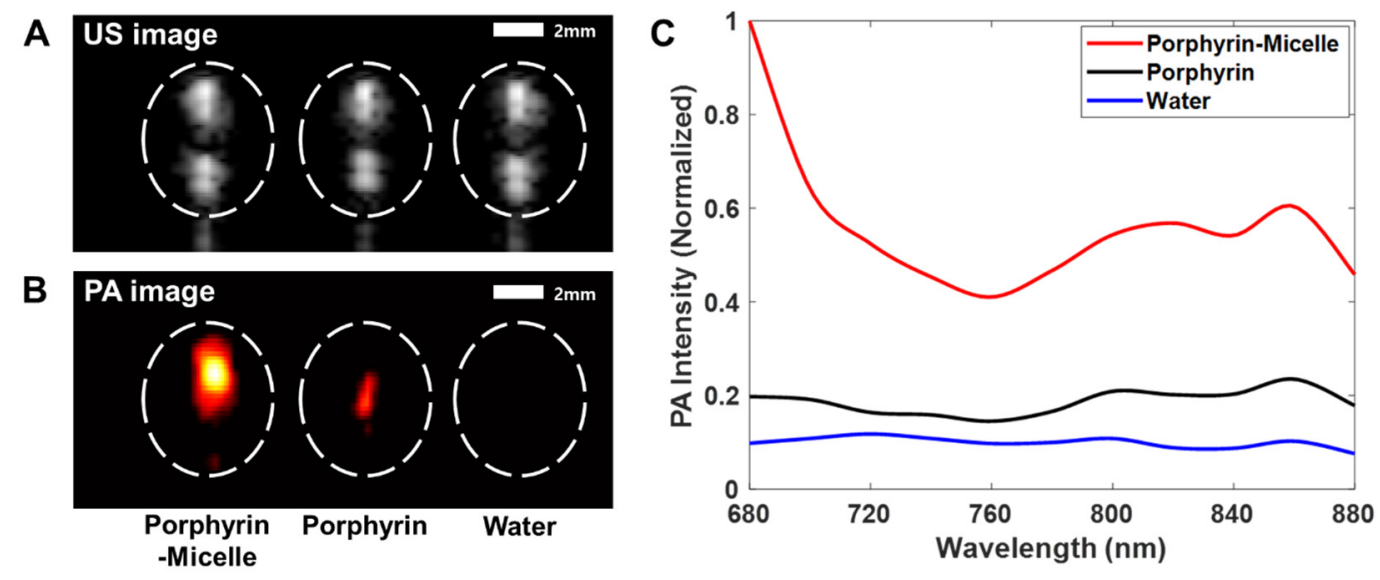

Figure 4. Ultrasound and photoacoustic images of silicone tubes containing Porphyrin-Micelle (left), Porphyrin (center), and water (right): (A) ultrasound images and (B) photoacoustic images. (C) Plot of changes in photoacoustic signal intensity as a function of laser wavelength.

\subsection{Photoacoustic Imaging of the Tumor In Vivo}

The in vivo imaging performance of each particle was evaluated with the mouse models bearing PC3 and LNCaP tumors. After injecting porphyrin, PM and PMP, both ultrasound and PA images were acquired every $5 \mathrm{~min}$ for $30 \mathrm{~min}$. For the PA imaging, the optimal laser wavelength of $680 \mathrm{~nm}$ was used. The combined ultrasound and PA images are shown in Figure 5 before the injection of PMP (left panel of Figure 5A), PM (the left panel of Figure 5B), or porphyrin (left panel of Figure 5C). The PA signals were observed only in the cutaneous region of the mice prior to the injection, but the generation of the PA signals was negligible within the tumor regions indicated by the dashed white circles in Figure 5. Five minutes after PMP injection, the PA signal strength increased significantly within the LNCaP tumor, but it did not change much within the PC3 tumor (see Figure 5A,D,E). No significant changes in PA signal strengths were also observed within the LNCaP and PC3 tumors after the PM and porphyrin injection. The average PA signal intensity was 13 times higher for PMP inside the LNCaP tumor than for PMP inside the PC3 tumor or for PM and porphyrin inside both LNCaP and PC3 tumors. The results implied that only PMP nanoparticles bind well PSMA, which is highly overexpressed by prostate (LNCaP) tumors. 


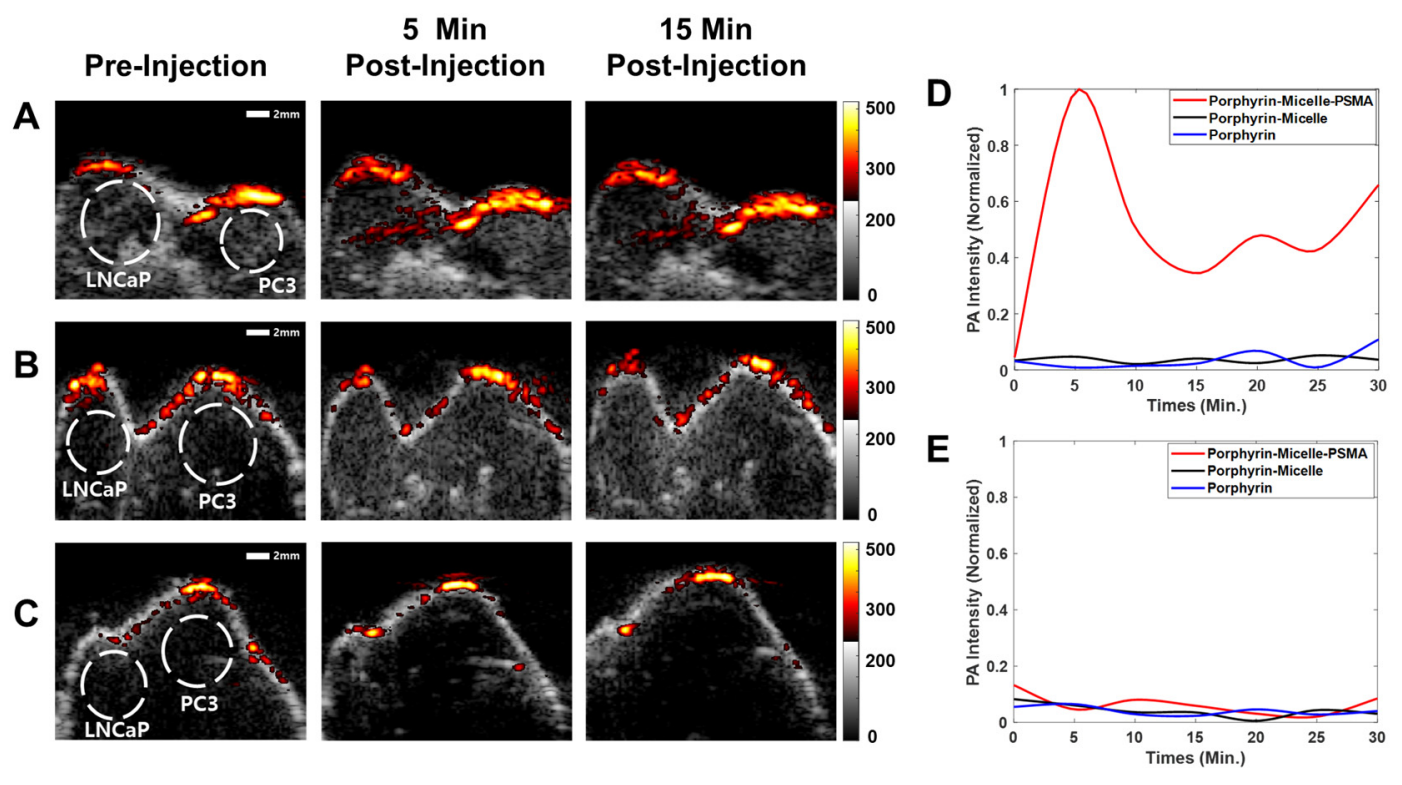

Figure 5. Combined ultrasound and photoacoustic images of the tumors in the mice before (left panels) and after intravenous injection of the particles (middle panels: after $5 \mathrm{~min}$, right panels: after $15 \mathrm{~min}$ ): (A) PMP, (B) PM, and (C) porphyrin. Plots of the changes in PA intensity inside (D) the LNCaP tumors and (E) the PC3 tumors as a function of time.

\section{Discussion}

Porphyrin-based materials are continuously being investigated in biomedicine owing to their unique characteristics, such as absorbing light efficiently at a wide range, thereby inducing chemical and physical changes. Because of these properties, porphyrin derivatives have been used in clinics as photodynamic agents in bladder cancer treatment. As demonstrated in this work, a higher accumulation of photosensitizers at the tumor region allows selective, highly sensitive PA imaging of the targeted tumors. To improve the circulation half-life and the accumulation of these PA agents, we also prepared porphyrins in nanoparticle formulations. The porphyrin micelles, PM, and the PSMA-targeting PMs (PMPs) were prepared in a way that porphyrins were packed inside the globular structure with branches of polyethylene glycol facing outward to the surface.

This phenomenon is presumed probably because the aggregated porphyrin concentration is higher than that of the porphyrin that only spread in all directions in the solution. In fact, when compared to blood, a signal about 4 times higher than that of only porphyrin was confirmed because of checking at the same concentration, and a superior signal was confirmed compared to only porphyrin. Since the concentration of PMP that can be injected is low compared to the high concentration of blood in living organisms, there was no noticeable difference in the in vivo experiment, but when PSMA attached to the PM, the signal difference between PM and PMP was secured in the LNCaP model. Through this, it was possible to confirm the possibility of PMP. In addition to its diagnostic use, PMP is expected to be used as an anticancer agent against the toxic side effects of anticancer drugs. According to the results of many studies on porphyrin, the possibility of cancer treatment was confirmed through ROS generated by the meeting of porphyrin and ultrasound, and it can be considered as an excellent particle not only for diagnosis through PMP but also used for cancer treatment using ultrasound in the future.

\section{Materials and Methods}

\subsection{Reagents and Equipment}

Hemin porphyrin was acquired from Sigma Aldrich (St. Louis, MO, USA). PEG3.5K was acquired from creative PEG Works (Chapel Hill, NC, USA) (Sodium borohydride $(\mathrm{NaBH} 4)$, sodium cyanoborohydride $(\mathrm{NaCNBH})$, Zolazepam $\left(\right.$ Zoletil $\left.^{\circledR}\right)$ was obtained 
fromVirbac (Virbac, Carros, France), and xylazine hydrochloride (Rompun 2\%)was acquired from Bayer (Bayer Korea, Seoul, Korea). The 1260 Infinity II LC system was acquired from Agilent Technologies (Agilent Technologies, Santa Clara, CA, USA).

\subsection{Preparation of $P M P$}

To synthesize PEG3.5K-TZ(PEG3.5K-methylenetetrazine), PEG3.5K-amine and methylene tetrazine-NHS were dissolved in DCM at 1:1 molar ratio over stirring for $30 \mathrm{~min}$. After DCM was evaporated by distillation, Hemin Porphyrin and DMF were added in the same batch and stirred for $1 \mathrm{~h}$. The solution was distilled, and the pellet was re-dispersed with distilled water. The solution was centrifugated at $15,000 \mathrm{rpm}$ for $15 \mathrm{~min}$ at $4{ }^{\circ} \mathrm{C}$ to discard unreacted water-insoluble material. The final material was checked with MALDI-TOF to see if the PM had been synthesized. The size of PM was measured with DLS and freezedried. To prepare PMP, PSMA targeting moiety, PM and CDI were dissolved in distilled water at 0.3:1:0.3 molar ratio over stirring for overnight. 5k Amicon was used to purify the PMP and stored at $5{ }^{\circ} \mathrm{C}$.

\subsection{Characterization of PMP}

The hydrodynamic size, polydispersity, and zeta potential of the prepared PMP and PM materials were measured by using the dynamic light scattering (DLS) (Zetasizer Nano ZS90; Malvern Instruments, Malvern, UK). The molecular weight of the synthesized PM was measured using MALDI-TOF and the morphology and size of the PMP and PM materials were further studied with transmission electron microscopy (TEM) and scanning electron microscopy (SEM) at the National Center for Inter-University Facilities, Seoul National University, Korea.

\subsection{Cell Culture}

Human prostate cancer line LNCaP and PC3 cells were acquired from the American Type Culture Collection (ATCC) and were cultured in Dulbecco's Modified Eagle Medium (DMEM) and Roswell Park Memorial Institute (RPMI), respectively, and supplemented with $10 \%$ heat-inactivated fetal bovine serum (FBS), $100 \mathrm{IU} / \mathrm{mL}$ penicillin, $100 \mathrm{mg} / \mathrm{mL}$ streptomycin, and $2 \mathrm{mM}$ L-glutamine. Cultures were stored in a humidified atmosphere with $5 \% \mathrm{CO}_{2}$ at $37^{\circ} \mathrm{C}$ and frequently tested for mycoplasma contamination. Cells were subcultured once they reached $80 \%$ confluence, determined by the trypan blue dye exclusion method.

\subsection{Cell Viability Assay}

The CellTiter $96^{\circledR}$ AQueous One Solution Cell Proliferation Assay (MTS) method was used to measure the effects of PM, PMP, and porphyrin on cell viability. LNcaP and PC3 cells were seeded on 96-well plates at a density of $5 \times 10^{3}$ cells per well and incubated overnight. First, the effects of PM, PMP, porphyrin on cell viability were evaluated by adding various concentrations to both LNCaP and PC 3 cells. Cells were removed from the incubator at certain times, and their viability was evaluated against the phosphate-buffered saline (PBS) controls using the MTS solution to derive approximate IC50 values.

\subsection{Confocal Laser Scanning Microscopy}

LNCaP and PC3 cells were seeded on 8-well chamber slides (Nunc-Lab-Tek-II Chamber Slide-System, Thermo-Fisher Scientific, Waltham, MA, USA) at a density of $3 \times 10^{4}$ cells per well and incubated overnight. On the next day, the cells were treated with various concentrations of Porphyrin, PM, and PMP and further incubated for varying periods. Once incubation was complete, the cells were fixed for $15 \mathrm{~min}$ with $4 \%$ formaldehyde and counter-stained with 40,6-diamidino-2-phenylindole dyes (DAPI, Thermo-Fisher Scientific, Waltham, MA, USA). During fixation and staining, the cells were washed with fresh PBS. The images were acquired using a confocal microscope (Carl Zeiss, Inc., Oberkochen, Germany), using the excitation/emission wavelengths of $600 \mathrm{~nm}$. 


\subsection{In Vivo Study}

Immunodeficient, 6-8 week-old nude female mice were purchased from Orient Bio (Seoul, Korea) for the toxicity and efficacy studies. The mice were acclimated for a week before the start of the study and were maintained at standard conditions in specific pathogen-free (SPF) environments: $25 \pm 2{ }^{\circ} \mathrm{C}$ temperature, $50 \pm 10 \%$ relative humidity, and $12 \mathrm{~h}$ light $/ 12 \mathrm{~h}$ dark. All mice were fed sterilized standard mouse chow and water ad libitum. After acclimatization, $1 \times 106 \mathrm{LNCaP}$ and PC3 cells suspended in Matrigel (Corning, Tewksbury, MA, USA) were injected into the right flank regions of the mice. Once the tumor volume had reached $\sim 150 \mathrm{~mm}^{3}$, the mice were randomly sorted for treatment. The tumor sizes were monitored with a digital caliper, and the volumes were calculated according to the formula width ${ }^{2} \times$ length $\times 0.5$. All the in vivo protocols (Approval Number: BA-1911-283-083-01) were verified according to the guidelines of the Seoul National University Bundang Hospital.

\subsection{Photoacoustic Protocols Ex-Vivo}

For ultrasound imaging and PA signal reception, a commercial ultrasound research imaging scanner (Vantage 128, Verasonics, Inc., Redmond, WA, USA) equipped with an ultrasound linear array transducer (L7-4, Verasonics Inc., Kirkland, WA, USA) was used. For PA imaging, the linear array transducer was integrated with custom-made bifurcated optical fibers. Laser pulses with a length of $7 \mathrm{~ns}$ were generated by a Nd:YAG laser excitation system Surelite III-10 and Surelite OPO Plus, Continuum Inc., Santa Clara, CA, USA) and delivered into the target regions through optical fibers. The laser pulse repetition rate was $10 \mathrm{~Hz}$ and the energy density was measured at $4.23 \mathrm{~mJ} / \mathrm{cm}^{2}$ in front of the optical fibers. Detailed experimental arrangement could be found in [41].

For optimal wavelength selection, three silicone tubes (AAQ04091, Tygon ${ }^{\circledR}$ MedicalTubing, Saint-Gobain Corp, Courbevoie, France) were prepared. The tube had an inner diameter of $1.27 \mathrm{~mm}$ (or 0.05 inches) and an outer diameter of $2.286 \mathrm{~mm}$ (or 0.09 inches). The tubes were immersed into a container filled with deionized water. Porphyrin, PM nanoparticles, and water were injected into the tubes. The concentrations of porphyrin and PM nanoparticles were each $0.8 \mathrm{mg} / \mathrm{mL}$. Ultrasound imaging scanning was conducted to place the tubes at the focal depth of the array transducer; the final location of the tubes was $25 \mathrm{~mm}$ from the array surface. PA signals were acquired by changing the laser wavelength from 680 to $880 \mathrm{~nm}$ in $10 \mathrm{~nm}$ increments. The stored ultrasound and PA signals were used to construct images on MATLAB (MathWorks Inc., Natick, MA, USA). The strength of PA signals inside the ultrasound images of the tubes was measured, and the maximum signal intensity was calculated.

The in vivo experiment was performed with the same imaging equipment, but the laser wavelength was fixed at $680 \mathrm{~nm}$, selected as the optimal wavelength. The ultrasound and PA images of both LNCaP and PC3 cells injected regions were acquired as reference images. The PMP, PM, and porphyrin were injected into the tumor sites of three mice, respectively. Both ultrasound and PA image data were acquired for $30 \mathrm{~min}$ at an interval of $5 \mathrm{~min}$ after the injection. The image data were used for construction of combined ultrasound and PA images with the MATLAB software. After delineating the LNCaP and PC3 tumor regions on the ultrasound images, the strengths of PA signals inside the regions were measured, and the average PA strength was calculated.

\section{Conclusions}

In this work, we synthesized porphyrin conjugated to PEG3.5K and clarified the hydrophilic and hydrophobic parts that induce self-assembled porphyrin micelles. In addition, by attaching a targeting moiety (PSMA), which can only be attached to LNCaP, the delivery also improved. Through the photoacoustic device, it was possible to confirm the increase in the signal of the substance and its binding affinity, and through this another possibility for prostate cancer diagnosis was confirmed. Here, if porphyrin and ROS 
generated by ultrasound are used together, it will be an excellent theragnostic material that can be used to diagnose and treat at the same time without using anticancer drugs.

Author Contributions: Conceptualization, D.K., W.H., J.H.C., and H.J.L.; formal analysis, D.K., W.H.; data curation, D.K., W.H.; writing-original draft preparation, D.K. and W.H.; writing-review and editing, D.K., and W.H.; supervision, J.H.C., H.J.L.; funding acquisition, H.J.L. All authors have read and agreed to the published version of the manuscript.

Funding: This research was supported by Basic Science Research Program through the National Research Foundation of Korea (NRF) funded by the Ministry of Education (NRF-2015R1D1A1A09056744), grant no 13-2019-0005 from the SNUBH Research Fund.

Institutional Review Board Statement: The study was approved by the Institutional Review Board (or Ethics Committee) of Seoul National University Bundang Hospital (BA-1911-283-083-01 and 2019-11-04).

Informed Consent Statement: Not applicable.

Data Availability Statement: N/A.

Conflicts of Interest: The authors declare no conflict of interest.

\section{References}

1. Nelen, V. Epidemiology of prostate cancer. In Prostate Cancer; Springer: New York, NY, USA, 2007; pp. 1-8.

2. Brawley, O.W. Trends in prostate cancer in the United States. J. Natl. Cancer Inst. 2012, 2012, 152-156. [CrossRef]

3. Chaudhry, P.; Asselin, E. Resistance to chemotherapy and hormone therapy in endometrial cancer. Endocr. Relat. Cancer 2009, 16, 363-380. [CrossRef] [PubMed]

4. Mannuel, H.D.; Hussain, A. Evolving role of surgery, radiation, hormone therapy, and chemotherapy in high-risk locally advanced prostate cancer. Clin. Genitourin. Cancer 2006, 5, 43-49. [CrossRef] [PubMed]

5. Dearnaley, D.P.; Khoo, V.S.; Norman, A.R.; Meyer, L.; Nahum, A.; Tait, D.; Yarnold, J.; Horwich, A. Comparison of radiation side-effects of conformal and conventional radiotherapy in prostate cancer: A randomised trial. Lancet 1999, 353, $267-272$. [CrossRef]

6. Korfage, I.J.; Hak, T.; de Koning, H.J.; Essink-Bot, M.-L. Patients' perceptions of the side-effects of prostate cancer treatment-A qualitative interview study. Soc. Sci. Med. 2006, 63, 911-919. [CrossRef] [PubMed]

7. Bottomley, D.; Ash, D.; Al-Qaisieh, B.; Carey, B.; Joseph, J.; Clair, S.S.; Gould, K. Side effects of permanent I125 prostate seed implants in 667 patients treated in Leeds. Radiother. Oncol. 2007, 82, 46-49. [CrossRef]

8. Dadfar, S.M.; Roemhild, K.; Drude, N.; von Stillfried, S.; Knüchel, R.; Kiessling, F.; Lammers, T. Iron oxide nanoparticles: Diagnostic, therapeutic and theranostic applications. Adv. Drug Deliv. Rev. 2019, 138, 302-325. [CrossRef]

9. Van der Leest, M.; Cornel, E.; Israël, B.; Hendriks, R.; Padhani, A.R.; Hoogenboom, M.; Zamecnik, P.; Bakker, D.; Yanti Setiasti, A.; Veltman, J.; et al. Head-to-head comparison of transrectal ultrasound-guided prostate biopsy versus multiparametric prostate resonance imaging with subsequent magnetic resonance-guided biopsy in biopsy-naive men with elevated prostate-specific antigen: A large prospective multicenter clinical study. Eur. Urol. 2019, 75, 570-578.

10. Hoeks, C.M.; Schouten, M.G.; Bomers, J.G.; Hoogendoorn, S.P.; de Kaa, C.A.H.-V.; Hambrock, T.; Vergunst, H.; Sedelaar, J.M.; Fütterer, J.J.; Barentsz, J.O. Three-tesla magnetic resonance-guided prostate biopsy in men with increased prostate-specific antigen and repeated, negative, random, systematic, transrectal ultrasound biopsies: Detection of clinically significant prostate cancers. Eur. Urol. 2012, 62, 902-909. [CrossRef]

11. Fascelli, M.; Rais-Bahrami, S.; Sankineni, S.; Brown, A.M.; George, A.K.; Ho, R.; Frye, T.; Kilchevsky, A.; Chelluri, R.; Abboud, S.; et al. Combined biparametric prostate magnetic resonance imaging and prostate-specific antigen in the detection of prostate cancer: A validation study in a biopsy-naive patient population. Urol. 2016, 88, 125-134. [CrossRef]

12. Park, B.K.; Park, J.W.; Park, S.Y.; Kim, C.K.; Lee, H.M.; Jeon, S.S.; Seo, S.I.; Jeong, B.C.; Choi, H.Y. Prospective evaluation of 3-T MRI performed before initial transrectal ultrasound-guided prostate biopsy in patients with high prostate-specific antigen and no previous biopsy. Am. J. Roentgenol. 2011, 197, W876-W881. [CrossRef]

13. Rais-Bahrami, S.; Siddiqui, M.M.; Vourganti, S.; Turkbey, B.; Rastinehad, A.R.; Stamatakis, L.; Truong, H.; Walton-Diaz, A.; Hoang, A.N.; Nix, J.W.; et al. Diagnostic value of biparametric magnetic resonance imaging (MRI) as an adjunct to prostate-specific antigen (PSA)-based detection of prostate cancer in men without prior biopsies. BJU Int. 2014, 115, 381-388. [CrossRef] [PubMed]

14. Siddique, S.; Chow, J.C.L. Application of Nanomaterials in biomedical imaging and cancer therapy. Nanomaterials 2020, 10, 1700. [CrossRef]

15. Siddique, S.; Chow, J.C.L. Gold nanoparticles for drug delivery and cancer therapy. Appl. Sci. 2020, 10, 3824. [CrossRef]

16. Labrie, F.; Dupont, A.; Suburu, R.; Cusan, L.; Tremblay, M.; Gomez, J.-L.; Emond, J. Serum prostate specific antigen as pre-screening test for prostate cancer. J. Urol. 1992, 147, 846-851. [CrossRef] 
17. Mohler, J.; Bahnson, R.R.; Boston, B.; Busby, J.E.; D’Amico, A.; Eastham, J.; Enke, C.A.; George, D.; Horwitz, E.M.; Huben, R.P.; et al. Prostate cancer. J. Natl. Compr. Cancer Netw. 2010, 8, 162-200. [CrossRef] [PubMed]

18. Govers, T.M.; Hessels, D.; Vlaeminck-Guillem, V.; Schmitz-Dräger, B.J.; Stief, C.G.; Martinez-Ballesteros, C.; Ferro, M.; BorqueFernando, A.; Rubio-Briones, J.; Sedelaar, J.P.M.; et al. Cost-effectiveness of SelectMDx for prostate cancer in four European countries: A comparative modeling study. Prostate Cancer Prostatic Dis. 2018, 22, 101-109. [CrossRef]

19. Evans, N.; Jackson, A.; Matlin, S.; Towill, R. High-performance liquid chromatographic analysis of porphyrins in clinical materials. J. Chromatogr. A 1976, 125, 345-355. [CrossRef]

20. Mang, T.S.; Dougherty, T.J.; Potter, W.R.; Boyle, D.G.; Somer, S.; Moan, J. Photobleaching of porphyrins used in photodynamic therapy and implications for therapy. Photochem. Photobiol. 1987, 45, 501-506. [CrossRef]

21. Kessel, D. Methods in Porphyrin Photosensitization; Springer: New York, NY, USA, 1985.

22. Akbarzadeh, I.; Yaraki, M.T.; Bourbour, M.; Noorbazargan, H.; Lajevardi, A.; Shilsar, S.M.S.; Heidari, F.; Mousavian, S.M. Optimized doxycycline-loaded niosomal formulation for treatment of infection-associated prostate cancer: An in-vitro investigation. J. Drug Deliv. Sci. Technol. 2020, 57, 101715. [CrossRef]

23. Yaraki, M.T.; Pan, Y.; Hu, F.; Yu, Y.; Liu, B.; Tan, Y.N. Nanosilver-enhanced AIE photosensitizer for simultaneous bioimaging and photodynamic therapy. Mater. Chem. Front. 2020, 4, 3074-3085. [CrossRef]

24. Bressler, N.M.; Bressler, S.B. Photodynamic therapy with verteporfin (Visudyne): Impact on ophthalmology and visual sciences. Investig. Ophthalmol. Vis. Sci. 2000, 41, 624-628.

25. Bressler, N.M. Photodynamic therapy of subfoveal choroidal neovascularization in age-related macular degeneration with verteporfin: Two-year results of 2 randomized clinical trials-tap report. Arch. Ophthalmol. 2001, 119, $198-207$.

26. Khurana, M.; Collins, H.A.; Karotki, A.; Anderson, H.L.; Cramb, D.T.; Wilson, B.C. Quantitative in vitro demonstration of two-photon photodynamic therapy using Photofrin ${ }^{\circledR}$ and Visudyne ${ }^{\circledR}$. Photochem. Photobiol. 2007, 83, 1441-1448. [CrossRef] [PubMed]

27. Zhou, Y.; Liang, X.; Dai, Z. Porphyrin-loaded nanoparticles for cancer theranostics. Nanoscale 2016, 8, 12394-12405. [CrossRef] [PubMed]

28. Zhang, L.; Lei, J.; Ma, F.; Ling, P.; Liu, J.; Ju, H. A porphyrin photosensitized metal-organic framework for cancer cell apoptosis and caspase responsive theranostics. Chem. Commun. 2015, 51, 10831-10834. [CrossRef] [PubMed]

29. Sternberg, E.D.; Dolphin, D.; Brückner, C. Porphyrin-based photosensitizers for use in photodynamic therapy. Tetrahedron 1998, 54, 4151-4202. [CrossRef]

30. Bonnett, R. Photosensitizers of the porphyrin and phthalocyanine series for photodynamic therapy. Chem. Soc. Rev. 1995, 24, 19-33. [CrossRef]

31. O'Connor, A.E.; Gallagher, W.; Byrne, A.T. Porphyrin and nonporphyrin photosensitizers in oncology: Preclinical and clinical advances in photodynamic therapy. Photochem. Photobiol. 2009, 85, 1053-1074. [CrossRef]

32. Maurer, T.; Eiber, M.; Schwaiger, M.E.M.; Gschwend, T.M.J.E. Current use of PSMA-PET in prostate cancer management. Nat. Rev. Urol. 2016, 13, 226-235. [CrossRef]

33. Ghosh, A.; Heston, W.D. Tumor target prostate specific membrane antigen (PSMA) and its regulation in prostate cancer. J. Cell. Biochem. 2004, 91, 528-539. [CrossRef] [PubMed]

34. Afshar-Oromieh, A.; Babich, J.W.; Kratochwil, C.; Giesel, F.L.; Eisenhut, M.; Kopka, K.; Haberkorn, U. The rise of PSMA ligands for diagnosis and therapy of prostate cancer. J. Nucl. Med. 2016, 57, 79S-89S. [CrossRef] [PubMed]

35. Ulrich, H.; Wrenger, C. Disease-specific biomarker discovery by aptamers. Cytom. Part A 2009, 75, 727-733. [CrossRef] [PubMed]

36. Dowling, P.; Clynes, M. Conditioned media from cell lines: A complementary model to clinical specimens for the discovery of disease-specific biomarkers. Proteomics 2011, 11, 794-804. [CrossRef] [PubMed]

37. Lolli, F.; Mulinacci, B.; Carotenuto, A.; Bonetti, B.; Sabatino, G.; Mazzanti, B.; D’Ursi, A.M.; Novellino, E.; Pazzagli, M.; Lovato, L.; et al. An N-glucosylated peptide detecting disease-specific autoantibodies, biomarkers of multiple sclerosis. Proc. Natl. Acad. Sci. USA 2005, 102, 10273-10278. [CrossRef] [PubMed]

38. Schmuck, S.; von Klot, C.A.; Henkenberens, C.; Sohns, J.M.; Christiansen, H.; Wester, H.-J.; Ross, T.L.; Bengel, F.M.; Derlin, T. Initial experience with volumetric 68Ga-PSMA I\&T PET/CT for Assessment of whole-body tumor burden as a quantitative imaging biomarker in patients with prostate cancer. J. Nucl. Med. 2017, 58, 1962-1968. [CrossRef]

39. Bravaccini, S.; Puccetti, M.; Bocchini, M.; Ravaioli, S.; Celli, M.; Scarpi, E.; De Giorgi, U.; Tumedei, M.M.; Raulli, G.; Cardinale, L.; et al. PSMA expression: A potential ally for the pathologist in prostate cancer diagnosis. Sci. Rep. 2018, 8, 1-8. [CrossRef] [PubMed]

40. Kang, J.; Kim, E.-K.; Kwak, J.Y.; Yoo, Y.; Song, T.-K.; Chang, J.H. Optimal laser wavelength for photoacoustic imaging of breast microcalcifications. Appl. Phys. Lett. 2011, 99, 153702. [CrossRef]

41. Kim, H.; Jo, G.; Chang, J.H. Ultrasound-assisted photothermal therapy and real-time treatment monitoring. Biomed. Opt. Express 2018, 9, 4472-4480. [CrossRef] 